\title{
Measurement of the cosmic ray all-particle and light-component energy spectra with the ARGO-YBJ experiment
}

\author{
I. De Mitri ${ }^{\mathrm{a}}$ on behalf of the ARGO-YBJ collaboration \\ Dipartimento di Matematica e Fisica "Ennio De Giorgi”, Università del Salento, Lecce, Italy \\ and Istituto Nazionale di Fisica Nucleare, INFN, Sezione di Lecce, Italy
}

\begin{abstract}
The ARGO-YBJ detector, located at high altitude in the Cosmic Ray Observatory of YangBaJing in Tibet (4300 m asl, about $600 \mathrm{~g} / \mathrm{cm}^{2}$ of atmospheric depth) provides the opportunity for the study, with unprecedented resolution, of cosmic ray physics in the primary energy region between $10^{12}$ and $10^{16} \mathrm{eV}$. Preliminary results of the measurements of the all-particle and light-component (i.e. protons and helium) energy spectra between approximately $5 \mathrm{TeV}$ and $5 \mathrm{PeV}$ are reported and discussed.
\end{abstract}

\section{Introduction}

There is a general consensus that galactic cosmic rays (hereafter CRs) up to the "knee" $(\sim 4 \mathrm{PeV}$, with $1 \mathrm{PeV}=$ $10^{15} \mathrm{eV}$ ) mainly originate in Supernova Remnants (SNRs). Recent measurements carried out by the balloon-borne CREAM experiment $[1,2]$ show that the proton and helium spectra from 2.5 to $250 \mathrm{TeV}$ are harder compared to lower energy measurements. As pointed out by several authors, the evolution of the proton and helium spectra and their subtle differences could be indications of the presence of different populations of $\mathrm{CR}$ sources contributing to the overall flux and operating in environments with different chemical compositions [3,4]. Diffusion effects during CR propagation in the Galaxy might also play an important role.

In the knee region (and above) the measurements of the CR primary spectrum are carried out by EAS arrays. In this case mass composition studies are extremely difficult and often affected by large systematic uncertainties. In the standard picture the average composition at the knee is dominated by light elements, and the knee itself is interpreted as the steepening of the $\mathrm{p}$ and He spectra [5].

However, different experimental results suggest a heavier composition at knee energies. For instance a hybrid measurement has been carried out by the EAS/TOP and MACRO experiments (by detecting, in coincidence, EAS Cherenkov light at $2000 \mathrm{~m}$ a.s.l. and underground muons below about $3000 \mathrm{~m}$ of water equivalent depth, respectively). The result implies a decreasing proton contribution to the primary flux well below the observed knee in the primary spectrum [6]. The same indication was previoulsy given by the analysis of the underground muon component alone by the MACRO experiment [7]. In addition, also the results of the Tibet AS $\gamma$ and the BASJE experiments, located at $4300 \mathrm{~m}$ a.s.1 and at $5200 \mathrm{~m}$ a.s.l. respectively, do favour a heavier composition because the proton component is no more dominant at the knee $[8,9]$.

a e-mail: ivan.demitri@le.infn.it
Indications for a substantial fraction of nuclei heavier than helium at $1 \mathrm{PeV}$ have also been obtained in measurements with delayed hadrons [10] as well as by the CASA-MIA collaboration [11].

A measurement of the CR primary energy spectrum (all-particle and light-component) in the energy range few $\mathrm{TeV}-10 \mathrm{PeV}$ is under way with the ARGO-YBJ experiment (for a description of the detector and a report of the latest physics results see [12]).

In order to cover this wide energy range, different approaches have been followed:

- "digital readout", based on the strip multiplicity, in the $5 \mathrm{TeV}-200 \mathrm{TeV}$ range [13];

- "analog readout", based on the particle density near the shower core, in the $100 \mathrm{TeV}-10 \mathrm{PeV}$ range;

- "hybrid measurement", carried out by ARGO-YBJ and a wide field of view Cherenkov telescope, in the $100 \mathrm{TeV}-\mathrm{PeV}$ region [14].

Preliminary results concerning the all-particle and the light-component (i.e. p+He) spectra obtained with the analog readout are summarized in the following. More details on the results obtained with the "hybrid measurement" are also given in $[14,15]$.

\section{Measurement of the all-particle spectrum}

The measurement of the CR energy spectrum up to $10 \mathrm{PeV}$ is under way exploiting the RPC charge readout of the ARGO-YBJ detector which allows studying the structure of the particle density distribution in the shower core region up to particle densities of about $10^{4} / \mathrm{m}^{2}$ [16].

The study of the charged particle lateral density function (LDF) at ground is expected to provide information on the longitudinal profile of the showers in the atmosphere, that is to estimate their development stage, or the so-called age, which is related to $X_{\max }$, the atmospheric depth at which the cascade reaches its

This is an Open Access article distributed under the terms of the Creative Commons Attribution License 4.0, which permits unrestricted use, distribution, and reproduction in any medium, provided the original work is properly cited. 


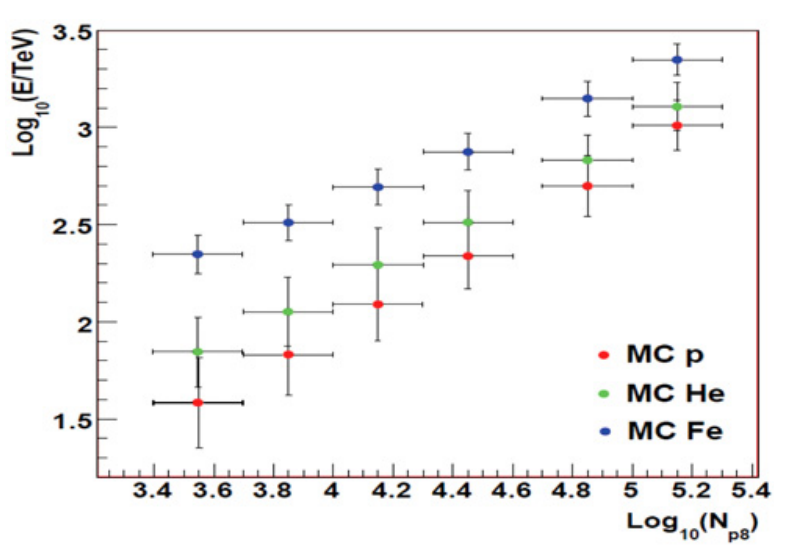

Figure 1. Primary energy as a function of the observed truncated size $\mathrm{N}_{p 8}$ for simulated showers due to different primary nuclei.

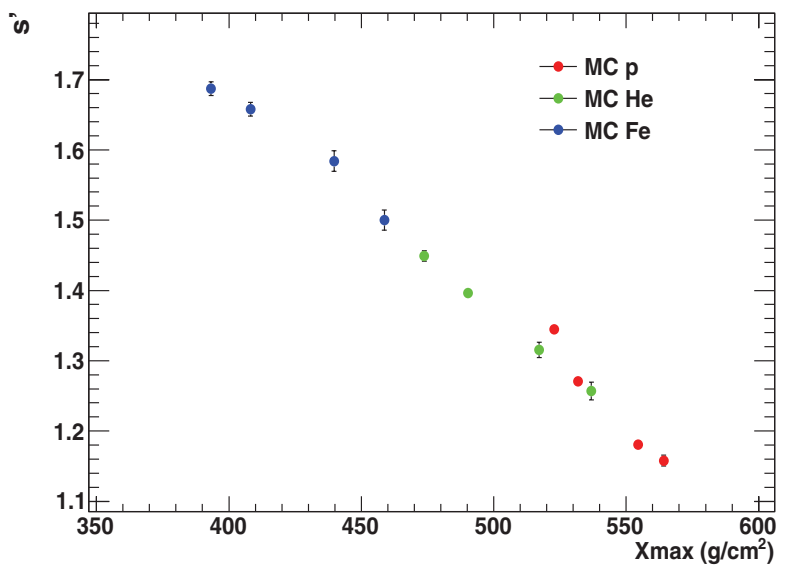

Figure 2. The age parameter $s^{\prime}$ resulting from the fit of the average LDF for simulated showers vs the corresponding $X_{\max }$ average values.

maximum size. This implies the possibility of selecting showers within given intervals of $X_{\max }$ or, equivalently, of $X_{d m}$, the grammage between the depth of the shower maximum and the detection level.

The shower development stage in the atmosphere, as observed at a fixed altitude (the detection one), depends on the energy of the interacting primary. For fixed energy, it depends on the nature of the primary: heavy primaries interact higher in the atmosphere, thus giving showers which, on average, reach their maximum at a larger distance from the detector than a lighter primary of the same energy. For this reason, the combined use of the shower energy and age estimations can ensure a sensitivity to the primary mass, thus giving the possibility of selecting a light $(\mathrm{p}+\mathrm{He})$ event sample with high efficiency.

Various observables were considered and analyzed in order to find a suitable estimator of the primary $\mathrm{CR}$ energy. Among them, according to $\mathrm{MC}$ simulations, $\mathrm{N}_{p 8}$, the number of particles detected within a distance of $8 \mathrm{~m}$ from the shower axis, resulted well correlated with energy, not biased by the finite detector size and not much affected by shower to shower fluctuations [17]. Therefore, the analysis is carried out in terms of different $\mathrm{N}_{p 8}$ intervals to select event samples corresponding to different primary energies. Nevertheless, as shown in Fig. 1, this truncated size is a mass-dependent energy estimator parameter.
In order to have a mass-independent parameter we fitted the LDFs of individual showers (up to $10 \mathrm{~m}$ from the core) event-by-event, for different $\mathrm{N}_{p 8}$ intervals and different shower initiating primaries, with a suitable function to get the shape parameter $s^{\prime}$ (see $[17,18]$ for details). From these studies we find that, for a given primary, the $s^{\prime}$ value decreases when $\mathrm{N}_{p 8}$ (i.e. the energy) increases, this being due to the observation of younger (deeper) showers at larger energies. Moreover, for a given range of $\mathrm{N}_{p 8}, s^{\prime}$ increases going from proton to iron, as a consequence of older (shallower) showers. Both dependencies are in agreement with the expectations, the slope $s^{\prime}$ being correlated with the shower age, thus reflecting its development stage. This outcome has two important implications, since the measurements of $s^{\prime}$ and $\mathrm{N}_{p 8}$ can both (i) help constraining the shower age and (ii) give information on the primary particle nature.

Concerning the first point, we show in Fig. 2 the $s^{\prime}$ values as obtained from the fit of the average LDFs, for each simulated primary type and $\mathrm{N}_{p 8}$ interval, as a function of the corresponding $X_{\text {max }}$ average value. As can be seen, the shape parameter $s^{\prime}$ depends only on the development stage of the shower, independently from the nature of the primary particle. That plot expresses an important universality of the LDF of detected EAS particles in terms of the lateral shower age. The LDF slope $s^{\prime}$ is a massindependent estimator of the average $X_{\max }$. This also implies the possibility to select most deeply penetrating showers (and quasi-constant $X_{d m}$ intervals) at different zenith angles, an important point for correlating the exponential angular rate distribution with the interaction length of the initiating particle [19]. Obviously showerto-shower fluctuations introduce unavoidable systematics, whose effects can be anyway quantified and taken into account.

Another implication is that $s^{\prime}$ from the LDF fit very close to the shower axis, together with the measurement of the truncated size $\mathrm{N}_{p 8}$, can give information on the primary particle nature, thus making possible the study of primary mass composition and the selection of a light-component data sample.

Assuming an exponential absorption after the shower maximum, we get $\mathrm{N}_{p 8}^{\max }$, a variable linearly correlated to the size at the shower maximum, by using $\mathrm{N}_{p 8}$ and $s^{\prime}$ measurements for each event and simply correcting with an exponential attenuation: $N_{p 8}^{\max } \approx N_{p 8} \cdot \exp \left[\left(h_{0} \sec \theta-\right.\right.$ $\left.\left.X_{\text {max }}\left(s^{\prime}\right)\right) / \lambda_{a b s}\right]$. A suitable choice of the absorption length $\lambda_{a b s}\left(=120 \mathrm{~g} / \mathrm{cm}^{2}\right)$ allows to get $\mathrm{N}_{p 8}^{\max }$, a parameter correlated with primary energy in an almost linear and mass independent way, providing an energy estimator with a $\log (\mathrm{E} / \mathrm{TeV}$ ) resolution of $0.10-0.15$ (getting better with energy).

As described in [16], the RPC charge readout system has 8 different and overlapping gain scale settings $(\mathrm{G} 0, \ldots ., \mathrm{G} 7$ from smaller to larger gains) in order to explore the particle density range $\approx 20-10^{4}$ particles $/ \mathrm{m}^{2}$. In this preliminary analysis the results obtained with two gain scales (so-called G1 and G4) are presented.

Selecting quasi-vertical events $\left(\theta<15^{\circ}\right)$ in terms of the truncated size $\mathrm{N}_{p 8}$ with the described procedure we reconstructed the $\mathrm{CR}$ all-particle energy spectrum shown 


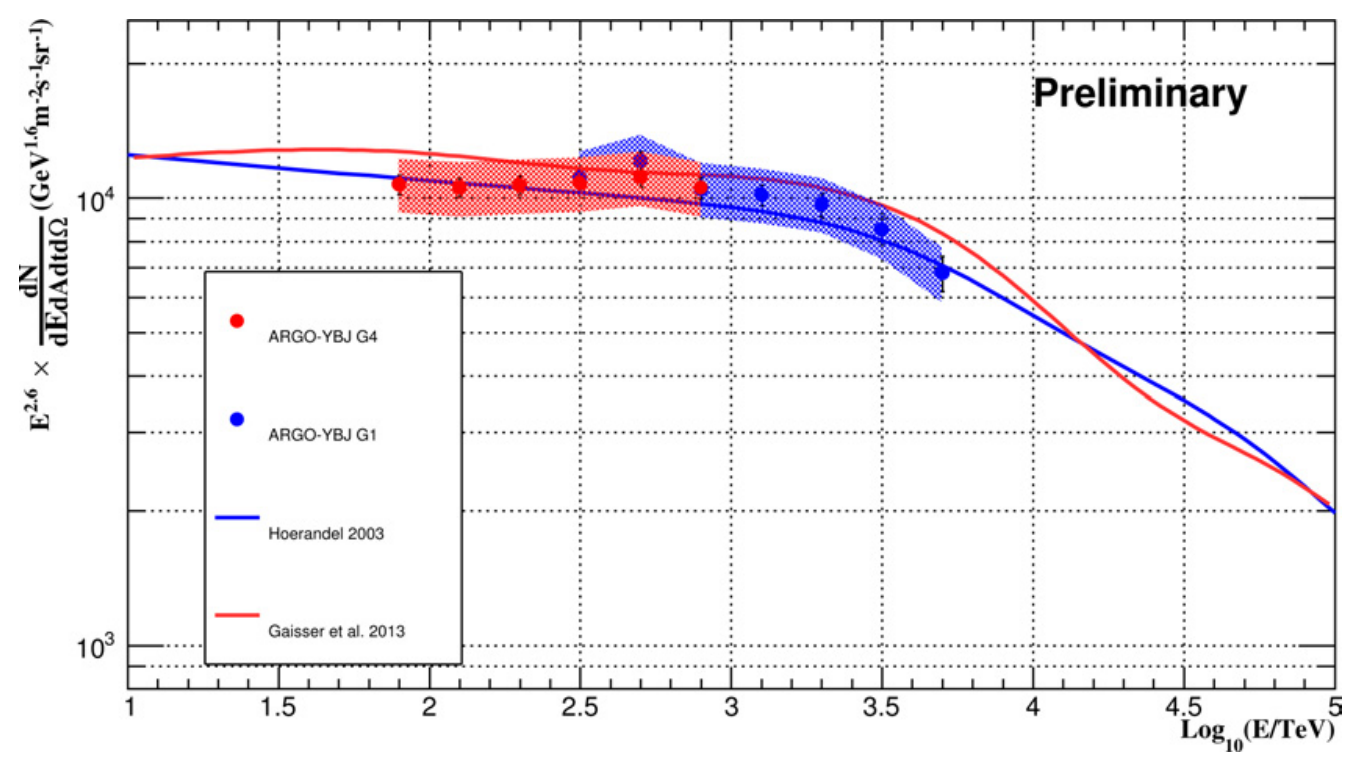

Figure 3. All-particle energy spectrum of primary CRs measured by ARGO-YBJ. Quasi-vertical events $\left(\theta<15^{\circ}\right)$ recorded with two different gain scales (G1 and G4) are plotted. The systematic uncertainty is shown by the shaded area and the statistical one by the error bars. The parametrizations provided by [20] and [3] are shown for comparison.
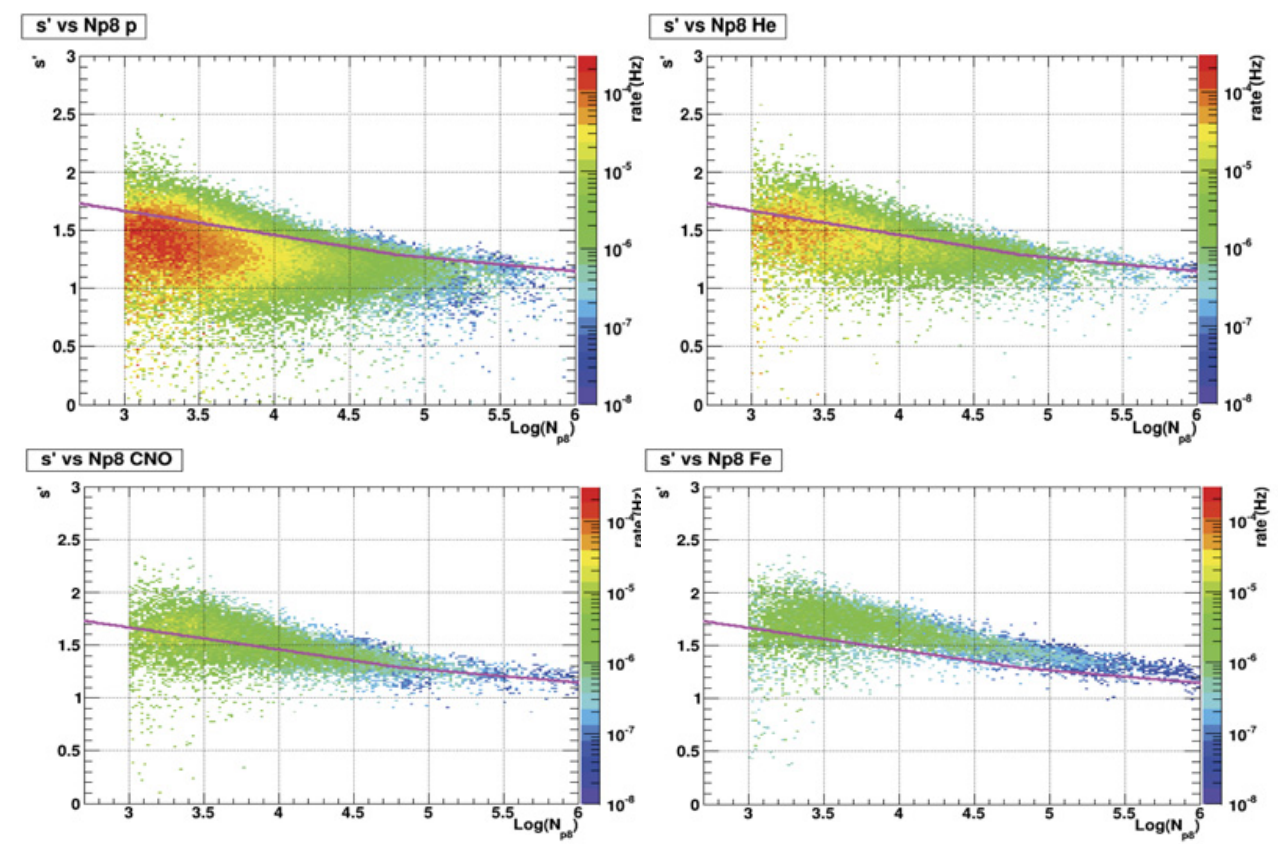

Figure 4. Relation between the LDF shape parameter $s^{\prime}$ and the truncated size $N_{p 8}$ for different nuclei. The p+He selection cut is shown by the lines.

in the Fig. 3 in the energy range $100-3000 \mathrm{TeV}$. In the plot a $\pm 14 \%$ systematic uncertainty, due to hadronic interaction models, selection criteria, unfolding algorithms, and aperture calculation, is shown by the shaded area. The statistical uncertainty is shown by the error bars. As can be seen from the figure, the two gain scales overlap making us confident about the event selection and the analysis procedure. The ARGO-YBJ all-particle spectrum is in fair agreement with the parametrizations provided by [20] and [3], showing evidence of a spectral index change at an energy consistent with the position of the knee. As shown in Fig. 6 this result is also consistent with previous measurements made by both direct and indirect experiments. This is also an important check on the absolute energy scale set for this analysis, whose systematic uncertainty has been anyhow conservatively estimated at the level of $10 \%$.

\section{Measurement of the light-component energy spectrum}

The CR light-component energy spectrum has been measured by ARGO-YBJ from about $5 \mathrm{TeV}$ to $500 \mathrm{TeV}$ [12-14]. The energy range is now being extended up to the few PeV region by using the RPC charge readout information and three different approaches. 


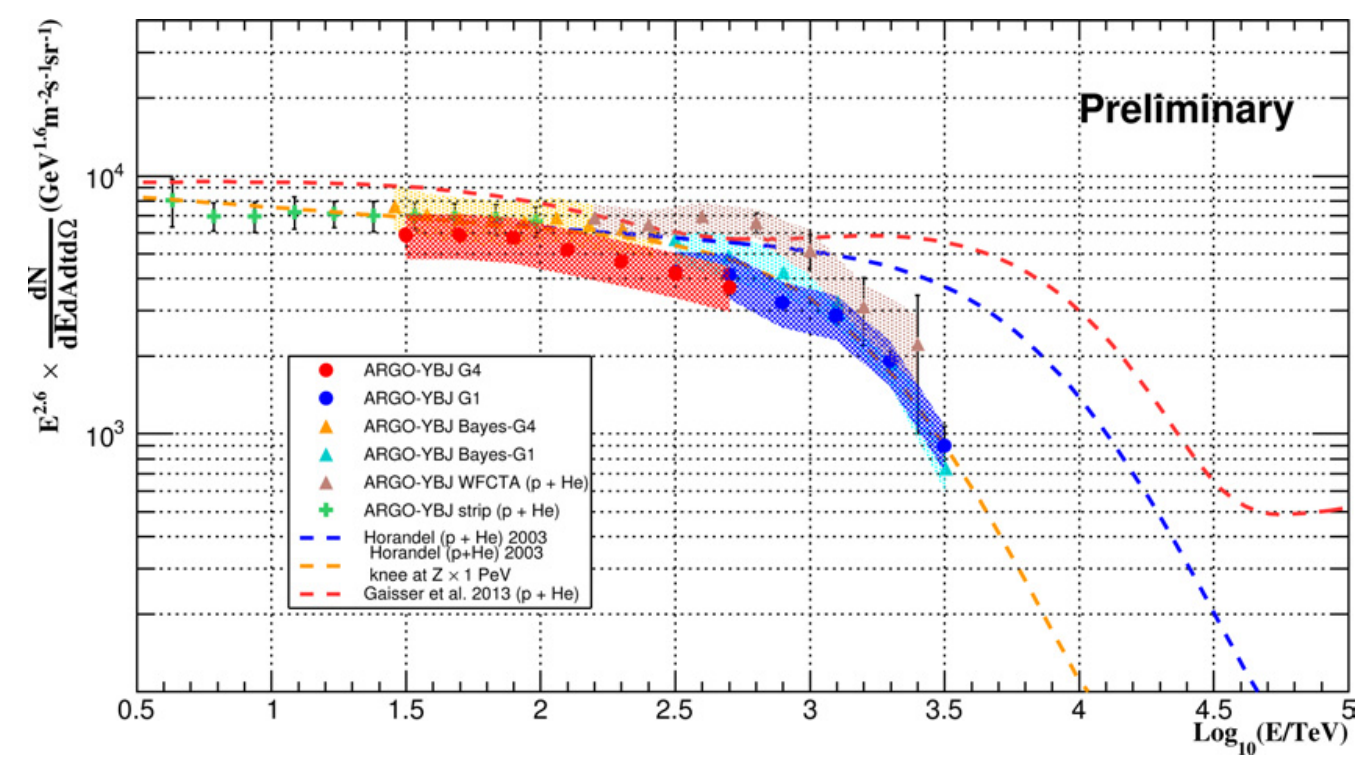

Figure 5. Light (i.e. p+He) component energy spectrum of primary CRs measured by ARGO-YBJ with four different analyses. Data recorded with two different gain scales (G1 and G4) are plotted. The systematic uncertainty is shown by the shaded area and the statistical one by the error bars. The parametrizations provided by [20] and [3] are shown for comparison. A Hörandel-like spectrum with a modified knee at $\mathrm{Z} \times 1 \mathrm{PeV}$ is also shown.

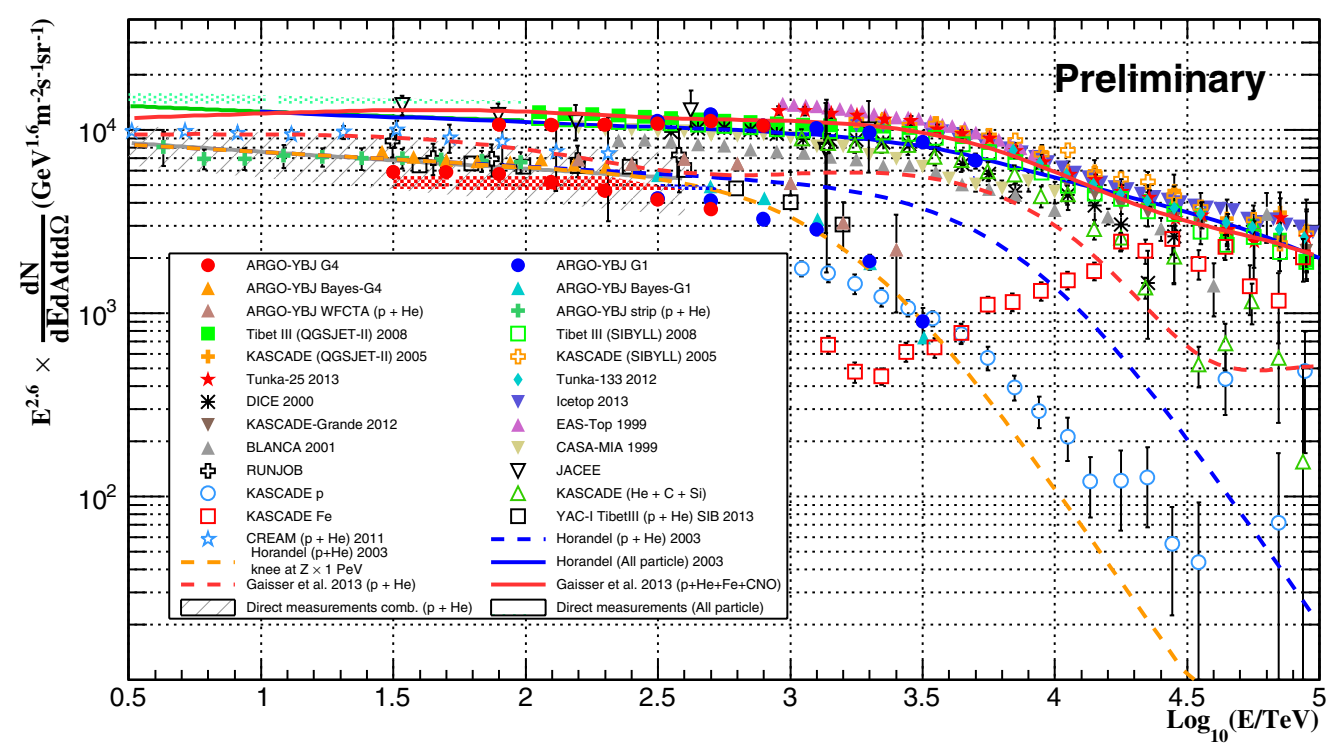

Figure 6. All particle and light $(\mathrm{p}+\mathrm{He})$ component energy spectra of primary CR measured by ARGO-YBJ and compared to different experimental results. The parametrizations provided by [20] and [3] are shown for comparison. A Hörandel-like spectrum with a modified knee at $\mathrm{Z} \times 1 \mathrm{PeV}$ is also shown.

(1) A selection of events in the $s^{\prime}-N_{p 8}$ space allowing to get a light-component sample of showers with a contamination of heavier nuclei less than about $15 \%$ (see Fig. 4).

(2) A Bayesian unfolding technique similar to that applied to measure the light-component spectrum up to $200 \mathrm{TeV}[12,13]$.

(3) The ARGO-YBJ/WFCTA hybrid measurement [14] with a different selection procedure which increases the aperture of a factor 2.4, thus allowing the extension to larger energies (see [15] for a detailed description of the method and a discussion of the results).

The preliminary results of the three analyses (and the previous ARGO-YBJ measurement below $200 \mathrm{TeV}$ ) are summarized in Fig. 5. The systematic uncertainty on the flux is shown by the shaded area and the statistical one by the error bars. A systematic uncertainty on the energy scale at the level of $5-10 \%$ (depending on the analysis) has also been conservatively estimated (not shown in the plots).

All three different analyses are consistent with low energy (direct) measurements and then show a clear evidence for a bending at larger energies but below $1 \mathrm{PeV}$. With respect to a single power-law with a spectral index -2.62 the deviation is observed at a level of about 6 s.d. The results obtained with the two analysis of RPC charge readout data (label 1 and 2 in the previous list) are in fair agreement. They also agree with the ARGO-YBJ/WFCTA hybrid measurement within systematic uncertainties and the possible difference in the energy scale. For comparison, 
the parametrizations of the light-component provided by [20] and [3] are shown by the blue and red dashed lines, respectively. A Hörandel-like spectrum with a modified knee at $\mathrm{Z} \times 1 \mathrm{PeV}$ is also shown (a factor four lower in energy than in the original formulation).

Finally the all particle and the light-component energy spectra measured by ARGO-YBJ are compared to a compilation of different experimental results in the Fig. 6.

\section{Conclusions}

The CR spectrum has been studied by the ARGO-YBJ experiment in a wide energy range $(\mathrm{TeVs} \rightarrow \mathrm{PeVs})$. This study is particularly interesting because not only it allows a better understanding of the so called "knee" of the energy spectrum and of its origin, but also provides a powerful cross-check among very different experimental techniques.

The all-particle spectrum (measured in the energy range $100 \mathrm{TeV}-3 \mathrm{PeV}$ ) is in good agreement with both theoretical parametrizations and previous measurements, making us confident about the selection and reconstruction procedures.

The light-component (i.e. $\mathrm{p}+\mathrm{He}$ ) has been reconstructed with high resolution up to about $5 \mathrm{PeV}$. The ARGO-YBJ preliminary result is in agreement with direct measurements and then show a clear indication of a bending below $1 \mathrm{PeV}$. Improvements of event selection with the full statistics and a complete analysis of systematic uncertainties is currently under way.

\section{References}

[1] H.S. Ahn et al., Astrophys. J. Lett. 714, L89 (2010)

[2] Y.S. Yoon et al., Astrophys. J. 728, 122 (2011)

[3] T.K. Gaisser, T. Stanev, S. Tilav, Front. Phys. 8(6) (2013) 748

[4] D. Caprioli et al., Astrop. Phys. 34, 447 (2011)

[5] W.D. Apel et al., Astrop. Phys. 31, 86 (2009)

[6] M. Aglietta et al., Astrop. Phys. 21, 223 (2004)

[7] M. Ambrosio et al. Phys. Rev. D 56 (1997) 1418

[8] M. Amenomori et al., Phys. lett. B 632, 58 (2006)

[9] H. Tokuno et al., Astrop. Phys. 29, 453 (2008)

[10] H. T. Freudenreich et al., Phys. Rev. D 41, 2732 (1990)

[11] M.A.K. Glasmacher et al., Astrop. Phys. 12, 1 (1999)

[12] I. De Mitri et al., Nucl. Instr. and Meth. in Phys. Res. A742 (2014) 2-9

[13] B. Bartoli et al., Phys. Rev. D 85, 092005 (2012)

[14] B. Bartoli et al., Chinese Phys. C 38, 045001 (2014)

[15] Z. Cao et al., Proc. of the Vulcano workshop 2014, Frascati Physics Series, in publication

[16] S. Mastroianni et al. in Proc. 33rd ICRC, (Rio de Janeiro, 2013) ID:1035

[17] I. De Mitri et al., in Proc. 33rd ICRC, (Rio de Janeiro, 2013) ID:0779

[18] A. D'Amone et al., in Proc. 33rd ICRC, (Rio de Janeiro, 2013) ID:0781

[19] G. Aielli et al., Phys. Rev. D 80, 092004 (2009)

[20] J.H. Hörandel, Astrop. Phys. 19, 193 (2003) 